\title{
Data-Centric Interactions on the Web
}

\author{
Paloma Díaz', Tim Hussein², Steffen Lohmann¹, and Jürgen Ziegler ${ }^{2}$ \\ ${ }^{1}$ Universidad Carlos III de Madrid, \\ Computer Science and Engineering Department, \\ Avenida de la Universidad 30, 28911 Leganés, Spain \\ \{pdp, slohmann\}@inf.uc3m.es \\ ${ }^{2}$ University of Duisburg-Essen, \\ Department of Computer Science and Applied Cognitive Science, \\ Lotharstrasse 65, 47048 Duisburg, Germany \\ \{tim.hussein, juergen.ziegler\}@uni-due.de
}

\begin{abstract}
The World Wide Web has become a global database in recent years, with an ever-growing amount of data that is published online every day. Interactions with this data raise a number of research questions and practical challenges that are not yet sufficiently investigated. These issues will be addressed by the International Workshop of Data-Centric Interactions on the Web. It will serve as a platform for researchers, developers, and designers to foster the exchange of experiences and to discuss novel research ideas and results regarding data-centric interactions on the web.
\end{abstract}

Keywords: Web interaction, user interfaces, data-centric systems, semantic web, visualization, large datasets, interactive systems, data management.

\section{Workshop Theme}

While the original World Wide Web was mainly a web of documents, today's web is characterized by an ever-growing amount of data that is published and linked in structured formats. Data about products or services is increasingly made available for public access by companies (e.g., Amazon A2S API, Google Maps API, etc.). Governments and organizations publish more and more often statistics and other data on the web, usually with the aim to increase transparency and empowering the public to utilize this data (e.g., data.gov, data.gov.uk, etc.). In other attempts, structured data is extracted from Wikipedia (e.g., DBpedia [1,2]) or from multiple web sources (e.g., Freebase [3, 4]). Last but not least, huge amounts of data and metadata are created by the web users themselves, either indirectly (e.g., via social tagging) or in directed community efforts (e.g., Open Directory Project, OpenStreetMap, etc.). These are just a few of the many examples where data is published on the web nowadays.

Several standards and best practices for the description, publication, linking, and exchange of web data have been developed in the past. Popular examples are $\mathrm{W} 3 \mathrm{C}$ specifications such as XML, SOAP, RSS, and RDF, the recommended best practices of the Linked Data initiative [5], and various vocabularies that emerged from these 
approaches (e.g., Dublin Core, SKOS, etc.). A similar line is taken in less formal attempts to structure data, such as microformats [6] or advanced tagging approaches (e.g., geotagging, hashtags, etc.).

However, the full potential of the data can only be exploited with well-designed user interfaces and powerful interaction techniques that allow an efficient exploration and utilization of the data. Although some early attempts to investigate the interaction with this data have been made in the past, there is still a large number of research questions and practical challenges that are not yet sufficiently addressed. Reoccurring interaction problems are differently solved and more general design recommendations and guidelines are just beginning to emerge. A need for reusable design patterns and interaction techniques as well as novel ideas, tools, and methods to present web data to the users is clearly recognizable. These and related issues of data-centric interactions on the web are addressed by the workshop.

\section{Format and Target Audience}

The workshop will be a full-day event consisting of paper presentations, discussions, tool demonstrations, and collaborative activities. It aims to bring together researchers, developers, and designers with a shared interest in data-centric interactions on the web. As several research areas are affected by the workshop theme, participants from various backgrounds and communities are expected to attend. The workshop will provide an interdisciplinary forum to exchange experiences and ideas and to discuss novel research results and future developments regarding data-centric interactions on the web.

The workshop will consist of 3-4 sessions, each focusing on a particular aspect of the workshop theme. Accepted papers will be presented in 15-20 minute time slots. A summarization of all papers at the end of each session will help to identify shared goals and challenges and stimulate an in-depth discussion of the session topics. One session will be dedicated to tool demonstrations and selected design cases. In a final wrap up session, open problems and a roadmap for future research efforts will be identified. Further information can be found on the workshop website at http://dciworkshop.org

\section{References}

1. Auer, S. Bizer, C., Kobilarov, G., Lehmann, J., Cyganiak, R., Ives, Z.: DBpedia: A Nucleus for a Web of Open Data. In: Proc. of ISWC 2006, LNCS, vol. 4825, pp. 722-735. Springer, Berlin/Heidelberg (2006)

2. DBpedia, http://dbpedia.org

3. Bollacker, K., Evans, C., Paritosh, P., Sturge, T., Taylor, J.: Freebase: a Collaboratively Created Graph Database for Structuring Human Knowledge. In: Proc. of SIGMOD 2008, pp. 1247-1250, ACM, New York (2008)

4. Freebase, http://www.freebase.com

5. Linked Data - Connect Distributed Data across the Web, http://linkeddata.org

6. Microformats, http://microformats.org/ 